ESAIM: COCV 21 (2015) 348-358

DOI: $10.1051 / \mathrm{cocv} / 2014018$
ESAIM: Control, Optimisation and Calculus of Variations

www.esaim-cocv.org

\title{
SHAPE DERIVATIVE OF THE CHEEGER CONSTANT
}

\author{
ENEA PARINi ${ }^{1}$ AND NicOlas SAINTIER ${ }^{2,3}$
}

\begin{abstract}
This paper deals with the existence of the shape derivative of the Cheeger constant $h_{1}(\Omega)$ of a bounded domain $\Omega$. We prove that if $\Omega$ admits a unique Cheeger set, then the shape derivative of $h_{1}(\Omega)$ exists, and we provide an explicit formula. A counter-example shows that the shape derivative may not exist without the uniqueness assumption.
\end{abstract}

Mathematics Subject Classification. 49Q10, 49Q20.

Received November 28, 2013. Revised March 6, 2014.

Published online October 17, 2014.

\section{INTRODUCTION}

Let $\Omega \subset \mathbb{R}^{n}$ be a bounded domain. The Cheeger constant of $\Omega$ is defined as

$$
h_{1}(\Omega):=\inf _{E \subset \Omega} \frac{P\left(E ; \mathbb{R}^{n}\right)}{|E|} .
$$

Here $P\left(E ; \mathbb{R}^{n}\right)$ is the distributional perimeter of $E$ measured with respect to $\mathbb{R}^{n}$, while $|E|$ is the $n$-dimensional Lebesgue measure of $E$. A set $C \subset \Omega$ for which the infimum is attained is called a Cheeger set.

The problem of finding a Cheeger set for a given domain $\Omega$ has extensively received attention in the last decades, starting from the original work of Cheeger [5]. For an introductory survey on the Cheeger problem we refer to [18]; here we recall that for every bounded domain $\Omega$ with Lipschitz boundary there exists at least one Cheeger set. Uniqueness does not hold in general, but it is guaranteed if we assume $\Omega$ to be convex; in this case the Cheeger set turns out to be convex and of class $C^{1,1}$ (see [1]). The Cheeger constant can be obtained as the limit for $p \rightarrow 1$ of the first eigenvalue $\lambda_{p}(\Omega)$ of the $p$-Laplacian under Dirichlet boundary conditions (see [12]), and corresponds to the first eigenvalue of the 1-Laplacian (see [14]).

Shape analysis roughly consists in studying the regularity and the optimisation of a functional $J: \Omega \in \mathcal{A} \rightarrow$ $J(\Omega) \in \mathbb{R}$ defined over some class $\mathcal{A}$ of subsets $\Omega \subset \mathbb{R}^{n}$. Due to its physical relevance, a particularly important class of functionals are the ones defined in terms of the eigenvalues of some operator. A lot of works have been dedicated for instance to the study of the dependence of the eigenvalues of the Laplacian as functions of the

\footnotetext{
Keywords and phrases. Shape derivative, CHEEGER constant, 1-Laplacian.

1 LATP, Aix-Marseille Université, 39 rue Joliot Curie, 13453 Marseille cedex 13, France. enea.parini@univ-amu.fr

2 Instituto de Ciencias, University Nac. Gral Sarmiento, J. M. Gutierrez 1150, C.P. 1613 Los Polvorines, Pcia de Bs. As, Argentina

3 Dpto Matemática, FCEyN, University de Buenos Aires, Ciudad Universitaria, Pabellón I (1428) Buenos Aires, Argentina. nsaintie@dm.uba.ar; nsaintie@ungs.edu.ar
} 
domain under various boundary conditions. We refer for example to the monograph [11] for an introduction to the field of shape analysis.

In order to optimize $J$ over $\mathcal{A}$ it is important to determine how sensitive is $J$ under perturbation of a given set $\Omega$. Given a smooth vector field $V \in C_{c}^{\infty}\left(\mathbb{R}^{n} ; \mathbb{R}^{n}\right)$, define $F_{t}: \mathbb{R}^{n} \rightarrow \mathbb{R}^{n}$ as $F_{t}(x)=(I d+t V)(x)$. We then perturb $\Omega$ in the direction $V$ by considering the sets $\Omega_{t}=F_{t}(\Omega)$. The shape derivative of $J$ in the direction $V$ at $\Omega$ is then defined as

$$
J(\Omega, V)^{\prime}:=\lim _{t \rightarrow 0} \frac{J\left(\Omega_{t}\right)-J(\Omega)}{t} .
$$

For instance the shape derivative of the first eigenvalue $\lambda(\Omega)$ of the Laplacian with Dirichlet boundary condition is

$$
\lambda(\Omega, V)^{\prime}=-\int_{\partial \Omega}\left|\frac{\partial u}{\partial \nu}\right|^{2}\langle V, \nu\rangle \mathrm{d} \mathcal{H}^{n-1}
$$

where $u$ is the unique positive normalized eigenfunction in $\Omega$ and $\nu$ is the unit exterior normal to $\partial \Omega$. This formula has been generalized in $[8,16]$ to the first eigenvalue $\lambda_{p}(\Omega)$ of the $p$-Laplacian $(p>1)$ :

$$
\lambda_{p}(\Omega, V)^{\prime}=-(p-1) \int_{\partial \Omega}\left|\frac{\partial u_{p}}{\partial \nu}\right|^{p}\langle V, \nu\rangle \mathrm{d} \mathcal{H}^{n-1},
$$

where $u_{p}$ is the unique positive normalized eigenfunction in $\Omega$.

General results about the stability of the Cheeger constant $h_{1}(\Omega)$ as a function of $\Omega$ have been obtained in [10]. In particular the shape derivative was computed but only in the case $V(x)=\lambda x, \lambda \in \mathbb{R}$. The main purpose of this paper is to provide a formula for the shape derivative of $h_{1}(\Omega)$ in the case of an arbitrary deformation field $V$. Notice that setting $p=1$ formally in (1.1) does not give any meaningful information. Indeed it is known that characteristic functions of Cheeger sets are, up to a multiplicative constant, normalized first eigenfunctions of the 1-Laplacian and they are obtained as limit of eigenfunctions of the $p$-Laplacian as $p$ goes to 1 (see Sect. 2). Therefore, if $C$ is a Cheeger set, the normal derivative should be thought as equal to $-\infty$ on $\partial \Omega \cap \partial C$, so that the integral in (1.1) would be infinite. This kind of problem has also been considered in [20] where the shape derivative of the best Sobolev constant for the embedding of $B V(\Omega)$ into $L^{1}(\partial \Omega)$ was computed. Let us mention finally that the other extreme case $p=+\infty$ corresponding to the first eigenvalue of the $\infty$-Laplacian has been recently studied in $[7,17,19]$ for Dirichlet, Steklov and Neumann boundary condition respectively.

The main result of our paper is the following.

Theorem 1.1. Let $\Omega$ be a bounded Lipschitz domain. Let $V \in C_{c}^{\infty}\left(\mathbb{R}^{n} ; \mathbb{R}^{n}\right)$, and let $F_{t}: \mathbb{R}^{n} \rightarrow \mathbb{R}^{n}$ be the one-parameter family of diffeomorphisms defined by $F_{t}(x)=(I d+t V)(x)$. Set $\Omega_{t}=F_{t}(\Omega)$. Then

$$
\lim _{t \rightarrow 0} h_{1}\left(\Omega_{t}\right)=h_{1}(\Omega) .
$$

If moreover $\Omega$ admits a unique Cheeger set $C$ then the shape derivative

$$
h_{1}(\Omega, V)^{\prime}=\lim _{t \rightarrow 0} \frac{h_{1}\left(\Omega_{t}\right)-h_{1}(\Omega)}{t}
$$

exists and is given by

$$
h_{1}(\Omega, V)^{\prime}=\frac{1}{|C|} \int_{\partial^{*} C}\left(\operatorname{div}_{\partial C} V-h_{1}(\Omega)\langle V, \nu\rangle\right) \mathrm{d} \mathcal{H}^{n-1},
$$

where $\partial^{*} C$ is the reduced boundary of $C, \nu$ is the unit exterior normal vector on $\partial^{*} C$, and $\operatorname{div}_{\partial \Omega} V(x)=$ $\operatorname{div} V(x)-(\nu(x), D V(x) \nu(x)), x \in \partial^{*} \Omega$, is the tangential divergence of $V$ on $\partial \Omega$. 
In the case where $\partial C$ is of class $C^{1,1}$, this formula can be simplified:

Corollary 1.2. If $\Omega$ admits a unique Cheeger set $C$ and $\partial C$ is of class $C^{1,1}$, then the shape derivative of $h_{1}(\Omega)$ is given by the formula

$$
h_{1}(\Omega, V)^{\prime}=\frac{1}{|C|} \int_{\partial C \cap \partial \Omega}\left(\kappa-h_{1}(\Omega)\right)\langle V, \nu\rangle \mathrm{d} \mathcal{H}^{n-1},
$$

where $\kappa(x)=$ div $\nu$ is the sum of the principal curvatures of $\partial \Omega$ at the point $x$ (i.e. $(n-1)$ times the mean curvature), and $\nu$ is the unit exterior normal to $\partial \Omega$.

The assumption in the Corollary is in particular satisfied for every dimension $n$ when $\Omega$ is convex (see [1]), or in dimension $n \leq 7$ when $\partial \Omega$ is of class $C^{1,1}$ and admits a unique Cheeger set $C$ (see [4]). We point out that the uniqueness hypothesis is necessary. Indeed, at the end of this paper we provide a counter example of a domain admitting more than one Cheeger set, which is not shape differentiable for some choice of $V$. However, it is interesting to observe that the bounded domains $\Omega$ admitting a unique Cheeger set (and hence shape differentiable) are dense in the $L^{1}$ topology (see [4]).

\section{DEFINITIONS AND PRELIMINARY RESUlts}

Let $\Omega \subset \mathbb{R}^{n}$ be an open set. The total variation in $\Omega$ of a function $u \in L^{1}(\Omega)$ is defined as

$$
|D u|(\Omega):=\sup \left\{\int_{\Omega} u \operatorname{div} \varphi \mid \varphi \in C_{c}^{1}\left(\Omega ; \mathbb{R}^{n}\right),\|\varphi\|_{\infty} \leq 1\right\} .
$$

A function $u$ such that $|D u|(\Omega)<+\infty$ is said to be of bounded variation. The space of the functions of bounded variation will be denoted by $B V(\Omega)$. It can be easily proved that the total variation is lower semicontinuous with respect to the $L^{1}$-convergence (see [9]). Moreover, the following holds true. Suppose that $\Omega$ is a Lipschitz domain, and let $u \in B V(\Omega)$; if we denote by $\bar{u}$ the extension of $u$ by zero outside $\Omega$, then $\bar{u} \in B V\left(\mathbb{R}^{n}\right)$, and

$$
|D \bar{u}|\left(\mathbb{R}^{n}\right)=|D u|(\Omega)+\int_{\partial \Omega}|u| \mathrm{d} \mathcal{H}^{n-1}
$$

where $\mathcal{H}^{n-1}$ is the $(n-1)$-dimensional Hausdorff measure on $\partial \Omega$.

The perimeter of a set $E \subset \Omega$ (measured with respect to $\mathbb{R}^{n}$ ) is defined as

$$
P\left(E ; \mathbb{R}^{n}\right):=\left|D \chi_{E}\right|\left(\mathbb{R}^{n}\right),
$$

where $\chi_{E}$ is the characteristic function of $E$. The Cheeger constant of $\Omega$ is

$$
h_{1}(\Omega):=\inf _{E \subset \Omega} \frac{P\left(E ; \mathbb{R}^{n}\right)}{|E|},
$$

where $|E|$ stands for the $n$-dimensional Lebesgue measure of $E$. A Cheeger set is a set $C \subset \Omega$ such that

$$
\frac{P\left(C ; \mathbb{R}^{n}\right)}{|C|}=h_{1}(\Omega) .
$$

The existence of a Cheeger set for every bounded Lipschitz domain $\Omega$ is proved via the direct method of the Calculus of Variations. Uniqueness does not hold in general; however, any convex body has a unique Cheeger set (see [1]). If $C$ is a Cheeger set for $\Omega$, then $\partial C \cap \Omega$ is analytic, up to a closed singular set of Hausdorff dimension $n-8$; at the regular points of $\partial C \cap \Omega$, the mean curvature is equal to $\frac{h_{1}(\Omega)}{n-1}$ (see e.g. [18], Prop. 4.2). Morever, if $\partial \Omega$ is of class $C^{1,1}$, then also $\partial C$ enjoys the same regularity (see [4]); the same result holds if $\Omega$ is convex, as a consequence of the results in [21]. 
As an application of the coarea formula, $h_{1}(\Omega)$ can also be obtained as

$$
h_{1}(\Omega)=\inf _{u \in B V(\Omega) \backslash\{0\}} \frac{|D \bar{u}|\left(\mathbb{R}^{n}\right)}{\|u\|_{1}}
$$

or equivalently

$$
h_{1}(\Omega)=\inf \left\{|D \bar{u}|\left(\mathbb{R}^{n}\right) \mid u \in B V(\Omega),\|u\|_{1}=1\right\} .
$$

Therefore, $h_{1}(\Omega)$ can be seen as the first eigenvalue of the 1-Laplacian with Dirichlet boundary condition, which is defined formally as

$$
\Delta_{1} u=\operatorname{div}\left(\frac{\nabla u}{|\nabla u|}\right)
$$

and the characteristic functions of Cheeger sets are corresponding eigenfunctions. We refer to [14] for a thorough analysis of this problem. Here we observe that if $\Omega$ admits a unique Cheeger set $C$, then $u=\frac{1}{|C|} \chi_{C}$ is the unique nonnegative normalized eigenfunction of the 1-Laplacian, since every level set of a first eigenfunction is a Cheeger set (see [3], Thm. 2).

\section{Proof of the Main Results}

Recall that we are given a Lipschitz domain $\Omega \subset \mathbb{R}^{n}$ that we perturb in the direction of a smooth vector field $V \in C_{c}^{\infty}\left(\mathbb{R}^{n} ; \mathbb{R}^{n}\right)$ in the sense that we consider the perturbed domains

$$
\Omega_{t}=F_{t}(\Omega) \quad \text { with } \quad F_{t}(x)=(I d+t V)(x) .
$$

We let $h=h_{1}(\Omega)$ and $h_{t}=h_{1}\left(\Omega_{t}\right)$. We also assume that any function $u$ defined in $\Omega$ (resp. $\Omega_{t}$ ) is extended by 0 to $\mathbb{R}^{n} \backslash \bar{\Omega}$ (resp. $\mathbb{R}^{n} \backslash \overline{\Omega_{t}}$ ). With the notation of the previous section this means that $u=\bar{u}$.

We recall the change of variable formula for BV functions (see [9], Lem. 10.1). Let $G_{t}$ be the inverse of $F_{t}$ (which exists for small $t$ ). For an arbitrary function $u \in B V(\Omega)$, if we denote by $v$ the function of $B V\left(\Omega_{t}\right)$ defined by $v(x)=u\left(G_{t}(x)\right)$ we have the relations

$$
\int_{\Omega_{t}} v(x) \mathrm{d} x=\int_{\Omega} u(y)\left|\operatorname{det} D F_{t}(y)\right| \mathrm{d} y
$$

and

$$
|D v|\left(\mathbb{R}^{n}\right)=\int_{\mathbb{R}^{n}}\left|\left(D G_{t}\right)^{T} \sigma\right| \cdot\left|\operatorname{det} D F_{t}\right| \mathrm{d}|D u|,
$$

where $\sigma$ comes from the polar decomposition $D u=\sigma|D u|$.

Proof of Theorem 1.1. Let $u \in B V(\Omega)$ be a nonnegative eigenfunction for $h$ such that $\|u\|_{1}=1$ in the sense that $u$ is an extremal in (2) (which is known to exist). Consider the function $w_{t} \in B V\left(\Omega_{t}\right)$ defined as $w_{t}=u \circ G_{t}$. Then

$$
\left|D w_{t}\right|\left(\mathbb{R}^{n}\right)=\int_{\mathbb{R}^{n}}\left|\left(D G_{t}\right)^{T} \sigma\right| \cdot\left|\operatorname{det} D F_{t}\right| \mathrm{d}|D u|,
$$

where $\sigma$ comes from the polar decomposition $D u=\sigma|D u|$. Since $|\sigma|=1|\nabla u|-$ a.e., and $D F_{t} \rightarrow I d$ uniformly as $t \rightarrow 0$, so that $\left|\operatorname{det} D F_{t}\right| \rightarrow 1$ uniformly, we have using (2) and the above change of variable formula that

$$
h_{t} \leq \frac{\left|D w_{t}\right|\left(\mathbb{R}^{n}\right)}{\int_{\Omega_{t}} w_{t}}=\frac{\int_{\mathbb{R}^{n}}\left|\left(D G_{t}\right)^{T}\right| \cdot\left|\operatorname{det} D F_{t}\right| \mathrm{d}|D u|}{\int_{\Omega} u(y)\left|\operatorname{det} D F_{t}(y)\right| \mathrm{d} y}=(1+o(1)) \frac{\int_{\mathbb{R}^{n}} \mathrm{~d}|D u|}{\int_{\Omega} u(y) \mathrm{d} y} .
$$


It follows that

$$
\limsup _{t \rightarrow 0} h_{t} \leq h
$$

Let $u_{t} \in B V\left(\Omega_{t}\right)$ be a nonnegative extremal for $h_{t}$ such that $\left\|u_{t}\right\|_{1}=1$. Consider the function $v_{t} \in B V(\Omega)$ defined as $v_{t}=u_{t} \circ F_{t}$. Then

$$
\begin{aligned}
\left|D v_{t}\right|\left(\mathbb{R}^{n}\right) & =\int_{\mathbb{R}^{n}}\left|\left(D F_{t}\right)^{T} \sigma_{t}\right| \cdot\left|\operatorname{det} D G_{t}\right| \mathrm{d}\left|D u_{t}\right| \leq(1+o(1)) \int_{\mathbb{R}^{n}} \mathrm{~d}\left|D u_{t}\right| \\
& =(1+o(1)) h_{t} \\
& \leq h+o(1),
\end{aligned}
$$

and

$$
\int_{\Omega} v_{t} \mathrm{~d} x=\int_{\Omega_{t}} u_{t}\left|\operatorname{det} D F_{t}^{-1}\right| \mathrm{d} x=1+o(1) .
$$

Therefore $\left(v_{t}\right)$ is bounded in $B V\left(\mathbb{R}^{n}\right)$. Since the embedding of $B V\left(\mathbb{R}^{n}\right)$ into $L_{l o c}^{1}\left(\mathbb{R}^{n}\right)$ is compact, it follows that there exists a function $v \in B V\left(\mathbb{R}^{n}\right)$ such that (up to a subsequence), $v_{t} \rightarrow v$ a.e.. We deduce first that $v=0$ in $\mathbb{R}^{n} \backslash \Omega$, then, using (3.2), that

$$
\int_{\Omega} v \mathrm{~d} x=\lim _{t \rightarrow 0} \int_{\Omega} v_{t} \mathrm{~d} x=1
$$

and eventually according to (3.1), that

$$
|D v|\left(\mathbb{R}^{n}\right) \leq \liminf _{t \rightarrow 0}\left|D v_{t}\right|\left(\mathbb{R}^{n}\right) \leq h .
$$

Letting $v=v_{\mid \Omega}$, it follows that $\int_{\Omega} v \mathrm{~d} x=1$, and

$$
h \leq|D v|\left(\mathbb{R}^{n}\right) \leq \liminf _{t \rightarrow 0}\left|D v_{t}\right|\left(\mathbb{R}^{n}\right)=h .
$$

It follows that

$$
\lim _{t \rightarrow 0} h_{t}=h,
$$

and that $v$ is an extremal for $h$.

We assume from now on that $\Omega$ admits a unique Cheeger set $C \subset \Omega$. As a consequence, the only nonnegative normalized extremal for $h$ is $|C|^{-1} \chi_{C}$; this follows from the fact that every level set of an extremal is a Cheeger set (see [3], Thm. 2). In particular $u=v=|C|^{-1} \chi_{C}$. Therefore $v_{t} \rightarrow u$ in $L^{1}(\Omega)$ and

$$
\lim _{t \rightarrow 0}\left|D v_{t}\right|\left(\mathbb{R}^{n}\right)=|D u|\left(\mathbb{R}^{n}\right) .
$$

By [2], Proposition 3.13, this implies that

$$
\lim _{t \rightarrow 0} \int_{\mathbb{R}^{n}} \phi \mathrm{d}\left|D v_{t}\right|=\int_{\mathbb{R}^{n}} \phi \mathrm{d}|D u|
$$

for any $\phi \in C_{c}\left(\mathbb{R}^{n}\right)$.

Let us prove the differentiability. Using $w_{t}=u \circ G_{t}$ as a test-function for $h_{t}$, we obtain

$$
h_{t}-h \leq \frac{\int_{\mathbb{R}^{n}}\left|\left(D G_{t}\right)^{T} \sigma\right| \cdot\left|\operatorname{det} D F_{t}\right| \mathrm{d}|D u|}{\int_{\Omega} u(y)\left|\operatorname{det} D F_{t}(y)\right| \mathrm{d} y}-h .
$$

Observe that

$$
\left|\operatorname{det} D F_{t}(y)\right|=1+t \cdot \operatorname{div} V(y)+o(t),
$$


and

$$
\left|\left(D G_{t}(y)\right)^{T} \sigma(y)\right|=|\sigma(y)|-t\langle\sigma(y), D V(y) \cdot \sigma(y)\rangle+o(t),
$$

where $o(t)$ is uniform in $y$. Therefore

$$
\begin{aligned}
h_{t}-h \leq & \frac{h+t \int_{\mathbb{R}^{n}}(\operatorname{div} V-\langle\sigma, D V \sigma\rangle) \mathrm{d}|D u|+o(t)}{1+t \int_{\Omega} u \operatorname{div} V+o(t)}-h \\
= & \frac{t\left(\int_{\mathbb{R}^{n}}(\operatorname{div} V-\langle\sigma, D V \sigma\rangle) \mathrm{d}|D u|-h \int_{\Omega} u \operatorname{div} V\right)}{1+t \int_{\Omega} u \operatorname{div} V+o(t)} .
\end{aligned}
$$

We used the fact that $|\sigma|=1|D u|-$ a.e. and $u$ is a normalized extremal for $h$. It follows that

$$
\limsup _{t \rightarrow 0^{+}} \frac{h_{t}-h}{t} \leq \int_{\mathbb{R}^{n}}(\operatorname{div} V-\langle\sigma, D V \sigma\rangle) \mathrm{d}|D u|-h \int_{\Omega} u \operatorname{div} V
$$

and

$$
\liminf _{t \rightarrow 0^{-}} \frac{h_{t}-h}{t} \geq \int_{\mathbb{R}^{n}}(\operatorname{div} V-\langle\sigma, D V \sigma\rangle) \mathrm{d}|D u|-h \int_{\Omega} u \operatorname{div} V .
$$

Let us now prove the opposite inequality. We use $v_{t}$ as a test-function for $h$, and we obtain

$$
h_{t}-h=\int_{\mathbb{R}^{n}} \mathrm{~d}\left|D u_{t}\right|-h \geq \int_{\mathbb{R}^{n}}\left|\left(D G_{t}\right)^{T} \sigma_{t}\right| \cdot\left|\operatorname{det} D F_{t}\right| \mathrm{d}\left|D v_{t}\right|-\frac{\int_{\mathbb{R}^{n}} \mathrm{~d}\left|D v_{t}\right|}{\int_{\Omega} v_{t}},
$$

where $\sigma_{t}$ is such that $D u_{t}=\sigma_{t}\left|D u_{t}\right|$. We can also write

$$
h_{t}-h \geq \int_{\mathbb{R}^{n}} \mathrm{~d}\left|D v_{t}\right|+t \int_{\mathbb{R}^{n}}\left(\operatorname{div} V-\left\langle\sigma_{t}, D V \sigma_{t}\right\rangle\right) \mathrm{d}\left|D v_{t}\right|-\frac{\int_{\mathbb{R}^{n}} \mathrm{~d}\left|D v_{t}\right|}{\int_{\Omega} v_{t}}+o(t) .
$$

Since $\operatorname{div} V \in C_{c}\left(\mathbb{R}^{n}\right)$, we have

$$
\lim _{t \rightarrow 0} \int_{\mathbb{R}^{n}} \operatorname{div} V \mathrm{~d}\left|D v_{t}\right|=\int_{\mathbb{R}^{n}} \operatorname{div} V \mathrm{~d}|D u|
$$

Observe also that

$$
\int_{\Omega} v_{t}=1-t \int_{\mathbb{R}^{n}} u_{t} \operatorname{div} V+o(t)=1-t \int_{\mathbb{R}^{n}} u \operatorname{div} V+o(t)
$$

so that

$$
\begin{aligned}
\frac{\int_{\mathbb{R}^{n}} \mathrm{~d}\left|D v_{t}\right|}{\int_{\Omega} v_{t}} & =\int_{\mathbb{R}^{n}} \mathrm{~d}\left|D v_{t}\right|+t\left(\int_{\mathbb{R}^{n}} \mathrm{~d}\left|D v_{t}\right|\right)\left(\int_{\Omega} u \operatorname{div} V\right)+o(t) \\
& =\int_{\mathbb{R}^{n}} \mathrm{~d}\left|D v_{t}\right|+t h \int_{\Omega} u \operatorname{div} V+o(t),
\end{aligned}
$$

where we used the fact that $\left|D v_{t}\right|\left(\mathbb{R}^{n}\right)=h+o(1)$. Hence,

$$
h_{t}-h \geq t\left(\int_{\mathbb{R}^{n}} \operatorname{div} V \mathrm{~d}|D u|-h \int_{\Omega} u \operatorname{div} V-\int_{\mathbb{R}^{n}}\left\langle\sigma_{t}, D V \sigma_{t}\right\rangle \mathrm{d}\left|D v_{t}\right|\right)+o(t)
$$


Since $D v_{t} \rightarrow^{*} D u$ and $\left|D v_{t}\right|\left(\mathbb{R}^{n}\right) \rightarrow|D u|\left(\mathbb{R}^{n}\right)$, we have, according to Reshetnyak's Theorem (see [2], Thm. 2.39), that

$$
\lim _{t \rightarrow 0} \int_{\mathbb{R}^{n}} f\left(x, \sigma_{t}(x)\right) \mathrm{d}\left|D v_{t}\right|=\int_{\mathbb{R}^{n}} f(x, \sigma(x)) \mathrm{d}|D u| \quad \text { for any } f \in C_{b}\left(\mathbb{R}^{n} \times S^{n-1}\right) .
$$

It follows in particular that

$$
\lim _{t \rightarrow 0} \int_{\mathbb{R}^{n}}\left\langle\sigma_{t}, D V \sigma_{t}\right\rangle \mathrm{d}\left|D v_{t}\right|=\int_{\mathbb{R}^{n}}\langle\sigma, D V \sigma\rangle \mathrm{d}|D u|
$$

We thus obtain

$$
\limsup _{t \rightarrow 0^{+}} \frac{h_{t}-h}{t} \geq \int_{\mathbb{R}^{n}}(\operatorname{div} V-\langle\sigma, D V \sigma\rangle) \mathrm{d}|D u|-h \int_{\Omega} u \operatorname{div} V
$$

and

$$
\liminf _{t \rightarrow 0^{-}} \frac{h_{t}-h}{t} \leq \int_{\mathbb{R}^{n}}(\operatorname{div} V-\langle\sigma, D V \sigma\rangle) \mathrm{d}|D u|-h \int_{\Omega} u \operatorname{div} V .
$$

Therefore

$$
h_{1}(\Omega, V)^{\prime}=\lim _{t \rightarrow 0^{+}} \frac{h_{t}-h}{t}
$$

exists, and

$$
h_{1}(\Omega, V)^{\prime}=\int_{\mathbb{R}^{n}}(\operatorname{div} V-\langle\sigma, D V \sigma\rangle) \mathrm{d}|D u|-h \int_{\Omega} u \operatorname{div} V .
$$

Since $u=|C|^{-1} \chi_{C}$, we have that $|D u|=|C|^{-1} \mathcal{H}_{\mid \partial^{*} C}^{n-1}$ as a measure. We can thus rewrite the previous formula as

$$
\begin{aligned}
h_{1}(\Omega, V)^{\prime} & =\frac{1}{|C|}\left(\int_{\partial^{*} C}(\operatorname{div} V-\langle\sigma, D V \sigma\rangle) \mathrm{d} \mathcal{H}^{n-1}-h \int_{C} \operatorname{div} V\right) \\
& =\frac{1}{|C|} \int_{\partial^{*} C}(\operatorname{div} V-\langle\sigma, D V \sigma\rangle-h\langle V, \nu\rangle) \mathrm{d} \mathcal{H}^{n-1},
\end{aligned}
$$

where $\nu$ is the unit exterior normal to $\partial^{*} C$, and $\sigma$ is given by $D u=\sigma|D u|$. We observe that $\sigma=-\nu \mathcal{H}^{n-1}-$ a.e. on $\partial^{*} C$. Recall that

$$
\operatorname{div} V(x)-(\nu(x), D V(x) \nu(x))=\operatorname{div}_{\partial C} V(x), \quad x \in \partial^{*} C,
$$

is the tangential divergence of $V$ on $\partial^{*} C$ (see e.g. [11], Def. 5.4.6). We thus obtain that

$$
h_{1}(\Omega, V)^{\prime}=\frac{1}{|C|} \int_{\partial^{*} C}(\operatorname{div} \partial C V-h\langle V, \nu\rangle) \mathrm{d} \mathcal{H}^{n-1}
$$

which ends the proof of Theorem 1.1.

Proof of Corollary 1.2. Suppose that $\Omega$ admits a unique Cheeger set $C$ which is $C^{1,1}$. The unit exterior normal vector $\nu$ to $\partial C$ is thus defined at every point and is Lipschitz continuous. Its components are thus differentiable at $\mathcal{H}^{n-1}$ almost every point of $\partial C$; moreover, the quantity $\kappa:=\operatorname{div}_{\partial C} \nu$ belongs to $L^{\infty}(\partial C)$ and it can be seen as the distributional curvature of $\partial C$. Indeed one can easily adapt [11], Section 5.4.3 to the case of $C^{1,1}$ domains to obtain

$$
\operatorname{div}_{\partial C} V=\operatorname{div}_{\partial C} V_{\partial C}+\kappa(V, \nu) \quad \mathcal{H}^{n-1}-\text { a.e. },
$$

where $V_{\partial C}=V-(V, \nu) \nu$ is the tangential part of $V$, and

$$
\int_{\partial C} \operatorname{div} \partial C V_{\partial C} \mathrm{~d} \mathcal{H}^{n-1}=0 .
$$


Therefore it holds

$$
\int_{\partial C} \operatorname{div} \partial C=\int_{\partial C} \kappa\langle V, \nu\rangle
$$

and we can rewrite $(3.3)$ as

$$
\begin{aligned}
h_{1}(\Omega, V)^{\prime} & =\frac{1}{|C|} \int_{\partial C}\left(\operatorname{div}_{\partial C} V-h_{1}(\Omega)\langle V, \nu\rangle\right) \mathrm{d} \mathcal{H}^{n-1} \\
& =\frac{1}{|C|} \int_{\partial C}\left(\kappa-h_{1}(\Omega)\right)\langle V, \nu\rangle \mathrm{d} \mathcal{H}^{n-1} \\
& =\frac{1}{|C|} \int_{\partial C \cap \partial \Omega}\left(\kappa-h_{1}(\Omega)\right)\langle V, \nu\rangle \mathrm{d} \mathcal{H}^{n-1}
\end{aligned}
$$

since $\kappa=h_{1}(\Omega)$ in $\partial C \cap \Omega$. We then deduce (1.3).

We complete this section providing some explicit examples of computation of shape derivatives.

Example 3.1 (the ball). Let $\Omega=B_{R}$ be the ball of radius $R$, and $V$ is a vector field such that $V(x)=\nu(x)$ on $\partial B_{R}$, we have that $\frac{\mathrm{d} h_{t}}{\mathrm{~d} t}(0)=\left[\frac{d}{d r} h_{1}\left(B_{r}\right)\right](R)$. Since $h_{1}\left(B_{r}\right)=\frac{n}{r}$, we obtain using (1.3) that

$$
h_{1}(\Omega, V)^{\prime}=\frac{n \omega_{n} R^{n-1}}{\omega_{n} R^{n}} \cdot\left(\frac{n-1}{R}-\frac{n}{R}\right)=-\frac{n}{R^{2}}
$$

as expected. Now let $V$ be a volume-preserving perturbation; formula (1.3) becomes

$$
h_{1}(\Omega, V)^{\prime}=-\frac{1}{|\Omega|} \int_{\partial \Omega}\langle V, \nu\rangle \mathrm{d} \mathcal{H}^{n-1}=-\frac{1}{|\Omega|} \int_{\Omega} \operatorname{div} V=0
$$

in accordance with the well-known fact that the ball minimizes $h_{1}(\Omega)$ among all bounded domains with fixed volume.

Example 3.2 (The annulus). As another simple example take $\Omega=A_{r, R}=B_{R} \backslash \bar{B}_{r}$, the annulus $\{r<|x|<R\}$, $r<R$. According to $[6,13], A_{r, R}$ coincides with its Cheeger set so that

$$
h_{1}\left(A_{r, R}\right)=\frac{\left|\partial A_{r, R}\right|}{\left|A_{r, R}\right|}=n \frac{R^{n-1}+r^{n-1}}{R^{n}-r^{n}} .
$$

Taking $V(x)=\nu(x)$, we have by direct computation that

$$
\frac{\mathrm{d}}{\mathrm{d} t} h_{1}\left(A_{r-t, R+t}\right)_{\mid t=0}=n \frac{-R^{2 n-2}-r^{2 n-2}-(n-1) r^{n-2} R^{n}-(n-1) R^{n-2} r^{n}-2 n(r R)^{n-1}}{\left(R^{n}-r^{n}\right)^{2}},
$$

which coincides with formula (1.3):

$$
h_{1}(\Omega, V)^{\prime}=\left(\frac{n-1}{R}-h_{1}\left(A_{r, R}\right)\right) \frac{\left|\partial B_{R}\right|}{\left|A_{r, R}\right|}-\left(\frac{n-1}{r}+h_{1}\left(A_{r, R}\right)\right) \frac{\left|\partial B_{r}\right|}{\left|A_{r, R}\right|} .
$$

In dimension 2 this example can be generalized to curved annulus:

Example 3.3 (Curved annulus in the plane). Let $\Gamma$ be a smooth planar closed curve with no self-intersection, and $\Omega=\Sigma_{\Gamma, a}=\left\{x \in \mathbb{R}^{2}\right.$, dist $\left.(x, \Gamma)<a\right\}$ its tubular neighborhood of width $a$. We take $a$ so small that $\Omega$ has no self-intersection. According to [15], $h_{1}(\Omega)=\frac{1}{a}$ and $\Omega$ itself is the unique Cheeger set. We take $V=\nu$. Then $\Omega_{t}=\Sigma_{\Gamma, a+t}$ and $h(\Omega, V)^{\prime}=-\frac{1}{a^{2}}=-h_{1}(\Omega)^{2}$ which coincides with formula (1.3):

$$
h_{1}(\Omega, V)^{\prime}=\frac{1}{|\Omega|} \int_{\partial \Omega}\left(\kappa-h_{1}(\Omega)\right) \mathrm{d} \mathcal{H}^{n-1}
$$

since $\int_{\partial \Omega} \kappa=2 \pi \chi(\Omega)=0$ according to the Gauss-Bonnet formula. 
Example 3.4 (the square). We eventually provide an example where the Cheeger set is a proper subset of $\Omega$. According to [13] a rectangle $R_{a, b} \subset \mathbb{R}^{2}$ of edges $2 a$ and $2 b$ has a unique Cheeger set $C$ with

$$
h_{1}\left(R_{a, b}\right)=\frac{4-\pi}{2(a+b)-2 \sqrt{(a-b)^{2}+\pi a b}}
$$

(see e.g. one of the two squares in Fig. 1). We take $\Omega=[0,1] \times[0,1]=R_{1 / 2,1 / 2}$ and $V(x, y)=(\eta(x), 0)$ with $\eta: \mathbb{R} \rightarrow[0,1]$ smooth with compact support in $(1-\delta, 1+\delta), \delta$ small, and $\eta(x)=1$ for $x \in(1-\delta / 2,1+\delta / 2)$. Then $\Omega_{t}=(0,1+t) \times(0,1)$ for sufficiently small $t$. It follows by direct computations from (3.4) that

$$
h_{1}(\Omega, V)^{\prime}=-\frac{1}{2} h_{1}(\Omega)
$$

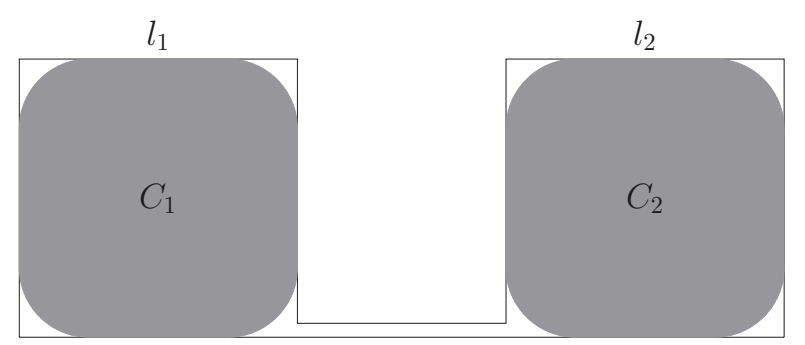

Figure 1. If $l_{1}=l_{2}$, the Cheeger sets are given by $C_{1}, C_{2}$ and $C_{1} \cup C_{2}$.

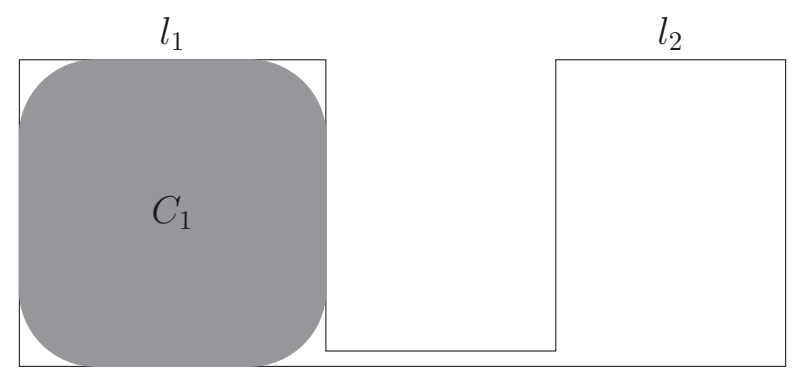

Figure 2. If $l_{1}>l_{2}$, the only Cheeger set is given by $C_{1}$.

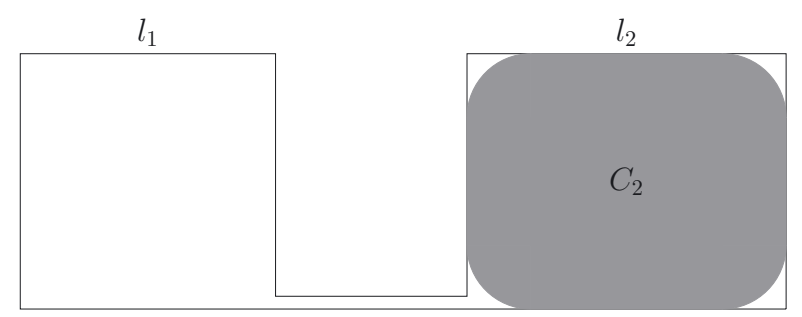

Figure 3 . If $l_{2}>l_{1}$, the only Cheeger set is given by $C_{2}$.

Since $\partial C \cap \Omega$ is made of arc of circle of radius $1 / h_{1}(\Omega)$, it is easily seen that

$$
\begin{gathered}
|C|=1-\frac{4-\pi}{h_{1}(\Omega)^{2}}=\frac{4 \sqrt{\pi}-2 \pi}{4-\pi}, \\
\mathcal{H}^{1}(\partial C \cap S)=1-\frac{2}{h_{1}(\Omega)}=\frac{2 \sqrt{\pi}-\pi}{4-\pi},
\end{gathered}
$$


where $S:=\{1\} \times[0,1]$. It follows that

$$
h_{1}(\Omega, V)^{\prime}=-h_{1}(\Omega) \frac{\mathcal{H}^{1}(\partial C \cap S)}{|C|},
$$

which is formula (1.3) since $\kappa=0$ on $\partial C \cap \partial \Omega,\langle V, \nu\rangle=1$ on $S$ and $\langle V, \nu\rangle=0$ on $\partial \Omega \backslash S$.

\section{A Counter-example to The DifFerentiability of $h_{1}(\Omega)$}

If $\Omega$ does not admit a unique Cheeger set, then $h_{1}(\Omega)$ is in general not differentiable. As a counter example, we consider the "barbell domain", made of two equal rectangles $R_{1}$ and $R_{2}$ linked by a thin strip (see Fig. 1), defined as

$$
\Omega=([0,1] \times[0,1]) \cup([1,2] \times[0, \varepsilon]) \cup([2,3] \times[0,1]),
$$

where $\varepsilon>0$ is sufficiently small. Let $V$ be a smooth vector field such that:

- $V$ is supported in $[3-\delta, 3+\delta] \times[-\delta, 1+\delta]$ for some small $\delta$;

- $V(x, y)=f(x, y) \overrightarrow{e_{1}}$ for some smooth nonnegative function $f$ satisfying $f(3, y)=1$ for $y \in[0,1]$.

In other words, $V$ shifts the far right edge of $\Omega$ to the right. For small positive values of $t, h_{1}\left(\Omega_{t}\right)$ behaves like the Cheeger constant of a rectangle obtained by enlarging $R_{2}$. Recalling formula (3.4) which gives the Cheeger constant of a rectangle $R_{a, b}$ of edges $2 a$ and $2 b$, we see that $\frac{\partial}{\partial b} h_{1}\left(R_{a, b}\right)<0$. Therefore

$$
\lim _{t \rightarrow 0^{+}} \frac{h_{1}\left(\Omega_{t}\right)-h_{1}(\Omega)}{t}<0 .
$$

For small negative values of $t, h_{1}\left(\Omega_{t}\right)=h_{1}\left(R_{1}\right)=h_{1}(\Omega)$ so that

$$
\lim _{t \rightarrow 0^{-}} \frac{h_{1}\left(\Omega_{t}\right)-h_{1}(\Omega)}{t}=0 .
$$

It follows that $h_{1}(\Omega)$ is not differentiable at $t=0$.

\section{REFERENCES}

[1] F. Alter and V. Caselles, Uniqueness of the Cheeger set of a convex body. Nonlin. Anal. 70 (2009) 32-44.

[2] L. Ambrosio, N. Fusco and D. Pallara, Functions of bounded variation and free discontinuity problems. Oxford University Press (2000).

[3] G. Carlier and M. Comte, On a weighted total variation minimization problem. J. Funct. Anal. 250 (2007) $214-226$.

[4] V. Caselles, A. Chambolle and M. Novaga, Some remarks on uniqueness and regularity of Cheeger sets, Rendiconti del Seminario Matematico della Università di Padova 123 (2010) 191-202.

[5] J. Cheeger, A lower bound for the smallest eigenvalue of the Laplacian, Problems in analysis: A symposium in honor of Salomon Bochner (1970) 195-199.

[6] F. Demengel, Functions locally almost 1-harmonic. Appl. Anal. 83 (2004) 865-896.

[7] J. Garcia-Azorero, J. Manfredi, I. Peral and J.D. Rossi, Steklov eigenvalues for the $\infty$-Laplacian. Atti Accad. Naz. Lincei Cl. Sci. Fis. Mat. Natur. Rend. Lincei Mat. Appl. 17 (2006) 199-210.

[8] J. Garcia Melian and J. Sabina De Lis, On the perturbation of eigenvalues for the p-Laplacian. C.R. Acad. Sci. Paris 332 (2001) 893-898.

[9] E. Giusti, Minimal surfaces and functions of bounded variation. In vol. 80 of Monogr. Math. Birkhäuser Verlag, Basel (1984).

[10] E. Hebey and N. Saintier, Stability and perturbations of the domain for the first eigenvalue of the 1-Laplacian. Arch. Math. 86 (2006) 340-351.

[11] A. Henrot and M. Pierre, Variation et optimisation de formes. Springer (2005).

[12] B. Kawohl and V. Fridman, Isoperimetric estimates for the first eigenvalue of the $p$-Laplace operator and the Cheeger constant. Comment. Math. Univ. Carolin. 44 (2003) 659-667.

[13] B. Kawohl and T. Lachand-Robert, Characterization of Cheeger sets for convex subsets of the plane. Pacific J. Math. 225 (2006) 103-118.

[14] B. Kawohl and F. Schuricht, Dirichlet problems for the 1-Laplace operator, including the eigenvalue problem. Commun. Contemp. Math. 9 (2007) 1-29. 
[15] D. Krejčiřík and A. Pratelli, The Cheeger constant of curved strips. Pacific J. Math. 254 (2011) 309-333.

[16] P.D. Lamberti, A differentiability result for the first eigenvalue of the $p$-Laplacian upon domain perturbation, Nonlinear analysis and Applications: to V. Lakshmikantham on his 80th birthday. Vol. 1, 2. Kluwer Acad. Publ., Dordrecht (2003) $741-754$.

[17] J.C. Navarro, J.D. Rossi, N. Saintier and A. San Antolin, The dependence of the first eigenvalue of the $\infty$-Laplacian with respect to the domain, Glasg. Math. J. 56 (2014) 241-249.

[18] E. Parini, An introduction to the Cheeger problem. Surveys Math. Appl. 6 (2011) 9-22.

[19] J.D. Rossi and N. Saintier, On the 1st eigenvalue of the $\infty$-Laplacian with Neumann boundary conditions, To appear in Houston $J$.

[20] N. Saintier, Estimates of the best Sobolev constant of the embedding of $B V(\Omega)$ into $L^{1}(\partial \Omega)$ and related shape optimization problems. Nonlinear Anal. 69 (2008) 2479-2491.

[21] E. Stredulinsky and W.P. Ziemer, Area minimizing sets subject to a volume constraint in a convex set. J. Geom. Anal. 7 (1997) 653-677. 